\title{
HIV infected adults do not have an excess of colonising bacteria in their large airways
}

\author{
Stephen B Gordon*1, Janelisa Musaya1, Lorna Wilson ${ }^{1}$, Amos Phiri ${ }^{1}$, \\ Eduard E Zijlstra $^{2}$ and Malcolm E Molyneux ${ }^{1}$
}

\author{
Address: ${ }^{1}$ Malawi-Liverpool-Wellcome Trust Research Programme, Universities of Malawi and Liverpool, PO Box 30096, Blantyre, Malawi and \\ ${ }^{2}$ Department of Medicine, University of Malawi College of Medicine, P O Box 360, Blantyre, Malawi \\ Email: Stephen B Gordon* - sgordon@mlw.medcol.mw; Janelisa Musaya - jmusaya@mlw.medcol.mw; \\ Lorna Wilson - lwilson@mlw.medcol.mw; Amos Phiri - aphiri@mlw.medcol.mw; Eduard E Zijlstra - eezijlstra@malawi.net; \\ Malcolm E Molyneux - mmolyneux@malawi.net \\ * Corresponding author
}

This article is available from: http://www.biomedcentral.com/I47I-2334/3/29

(C) 2003 Gordon et al; licensee BioMed Central Ltd. This is an Open Access article: verbatim copying and redistribution of this article are permitted in all media for any purpose, provided this notice is preserved along with the article's original URL.

\begin{abstract}
Background: HIV infected adults have increased susceptibility to bacterial pneumonia but the underlying immune defect is poorly understood. We tested the hypothesis that HIV infection might be associated with increased bacterial colonisation of distal airways by nasal flora, which would then predispose patients to bacterial pneumonia.
\end{abstract}

Methods: Healthy volunteer adults with normal chest radiographs were recruited. Bronchoscopy was carried out and uncontaminated mucosal samples were collected from proximal and distal sites in the large airways using a protected specimen brush. Samples were cultured to detect typical respiratory tract colonising organisms, and the proportion of samples found to contain colonising bacteria compared between HIV infected and uninfected subjects using non-parametric tests.

Results: Forty-nine subjects were studied of whom 27 were HIV infected. Colonising bacteria were identified in the nasopharynx of all subjects including Streptococcus pneumoniae in 6/49 subjects (5 HIV uninfected). Colonising bacteria were found in the distal airway of 6 subjects (3/27 HIV infected vs $3 / 22$ HIV uninfected; $\chi^{2}=0.07, p=0.8$ ). Streptococcus pneumoniae was identified in the trachea of all subjects with nasal colonisation but in the distal airway of only I subject.

Conclusions: There was no evidence to support a hypothesis of increased airway bacterial colonisation in healthy HIV infected subjects.

\section{Background}

Bacterial pneumonia [1] and invasive pneumococcal disease [2] are closely associated with HIV infection but the mechanisms underlying this increased susceptibility are poorly understood. Bed-bound patients develop an altered pattern of tracheo-bronchial colonizing flora in almost all cases, and this is thought to be a risk factor for nosocomial infection [3]. We hypothesized that HIV infection might lead to an increase in bronchial and bronchiolar bacterial colonization, which would increase the risk of bacterial pneumonia in these patients.

Protected specimen brushing (PSB) is a bronchoscopic technique used for obtaining lower respiratory tract material without contamination from upper respiratory tract colonising bacteria [4]. We used this technique in healthy 
volunteer adults to determine the rates of bacterial colonisation at different sites in the respiratory tract and the association of pulmonary colonisation with HIV infection. The expected rates of bacterial colonisation were found in HIV uninfected adults with no increase in rate in HIV infected adults, even with low CD4 counts.

\section{Methods}

Volunteers were recruited by advertisement in the Queen Elizabeth Central Hospital, Blantyre, Malawi. Healthy adults with normal chest radiographs were enrolled and tested for HIV using 2 rapid tests (HIV Serocard and HIV Gold, Trinity Biotech, Ireland). No subject had taken antibiotics within 2 weeks or any anti-retroviral medication.

Nasopharyngeal and oropharyngeal swabs were collected prior to bronchoscopy and directly inoculated on to blood agar plates. Bronchoscopy without sedation was carried out using topical lignocaine anaesthesia to the nasopharynx, larynx and trachea but not to the distal airways. After inspection to the carina only, a PSB catheter (Mill Rose microbiology brush, Keymed, UK) was directed in to the right middle lobe orifice. This catheter consists of an inner brush contained in a sterile sheath in turn itself contained within a wax-plugged outer sheath. The PSB catheter was passed to the point of mild resistance distally (at least $6 \mathrm{~cm}$ distal to the sub-segmental carina in the manner of a transbronchial biopsy) when the middle sheath and finally the sampling brush were advanced. After brushing, the brush was re-sheathed in reverse order, removed from the bronchoscope, reextruded and the brush cut off into $10 \mathrm{ml}$ of sterile saline. In the final 12 subjects, the PSB was directed to the distal right main bronchus (a much more proximal site and therefore likely to have more exposure to inhaled bacteria in all subjects) and sample collected as above followed by a $10 \mathrm{ml}$ tracheal washing collected in a sputum trap. All samples were processed in the laboratory within 20 minutes of the bronchoscopy.

In the laboratory, investigators were kept unaware of the HIV status of the particular patients being studied. Blood agar plates inoculated with upper airway swabs were inspected after 24 hours incubation at $37^{\circ} \mathrm{C}$ in candle jars and sub-cultured colonies used to identify colonising organisms. All liquid samples (distal and proximal PSB samples and tracheal washings) were vortexed vigorously and the brush removed when present. The saline was then directly sampled $(100 \mu \mathrm{l})$ on to culture plates (sheep blood agar, 15-lactose electrolyte-deficient (CLED), sabarose agar and chocolate blood agar) before being centrifuged and the residual pellet inoculated on to an identical set of plates. All plates were incubated for up to 5 days and cultured isolates were identified using standard methods including colony morphology, Gram stain, optochin sensitivity and coagulase testing.

The University of Malawi College of Medicine Research and Ethics Committee gave the study ethical approval and all volunteers gave written informed consent. A small remuneration approved by the Ethics Committee was given to all volunteers.

\section{Results}

49 volunteers ( 37 male, mean age 34 yrs; 12 female, mean age 23 yrs) were studied of whom 27 were HIV infected. CD4 counts were lower in HIV infected subjects (mean CD4 count of 243 cells/ul, range 7-706) compared to HIV uninfected subjects (mean CD4 count 721 cells/ul, range 444-1198). The HIV infected group had a mean body mass index (BMI) of $19.8 \mathrm{~kg} / \mathrm{m}^{2}$ (range 14.7-25) which was lower than that of the HIV uninfected group (mean BMI $21.3 \mathrm{~kg} / \mathrm{m}^{2}$; range $17.9-25.3$; ranksum test $\left.\mathrm{p}=0.03\right)$.

The percentage of subjects showing colonisation by a typical colonising organism at the different sites sampled is shown in Figure 1, divided according to HIV status. All subjects demonstrated either nasal or oral colonization or both, with a progressive reduction in colonisation distally. The organisms identified were Streptococcus viridans, beta-haemolytic Streptococcus spp., Streptococcus. pneumoniae, Staphylococcus aureus, Staphylococcus epidermidis, diptheroids and a single isolate of Aspergillus spp. Bacillus sp. and micrococci were discarded as contaminants as they are not typical of nasopharngeal isolates. No Neisseria meningitidis or Haemophilus influenzae were recovered. Bacterial isolates were all obtained from the vortexed specimen except in 1 distal lung sample where only the pellet grew Streptococcus sp. and Staphylococcus epidermidis.

There was no significant difference in the proportion of subjects with bacterial colonization at any site when compared by HIV serostatus (see Figure 1) and the only bacteria obtained from distal airways were Streptococcus viridans and Staphylococcus epidermidis recovered from 2 non-HIVinfected subjects. Grouping the findings to compare the proportions of subjects with bacteria detected above or below the carina by HIV status showed no significant difference (HIV negative 3/22; HIV positive $3 / 27: \chi^{2}$ test $\mathrm{p}=$ 0.8 ) and the mean CD 4 count of subjects with and without bacteria below the carina was not significantly different ( 472 cells/ul and 389 cells/ul; rank sum test $\mathrm{p}=0.5$ )

S. pneumoniae were detected in nasal or oral swabs from $6 /$ 49 subjects (5 HIV negative; 1 HIV positive) and in all tracheal washings from subjects with nasopharyngeal $S$. pneumoniae. S. pneumoniae was not detected in any distal airway sample but was recovered from the right main 


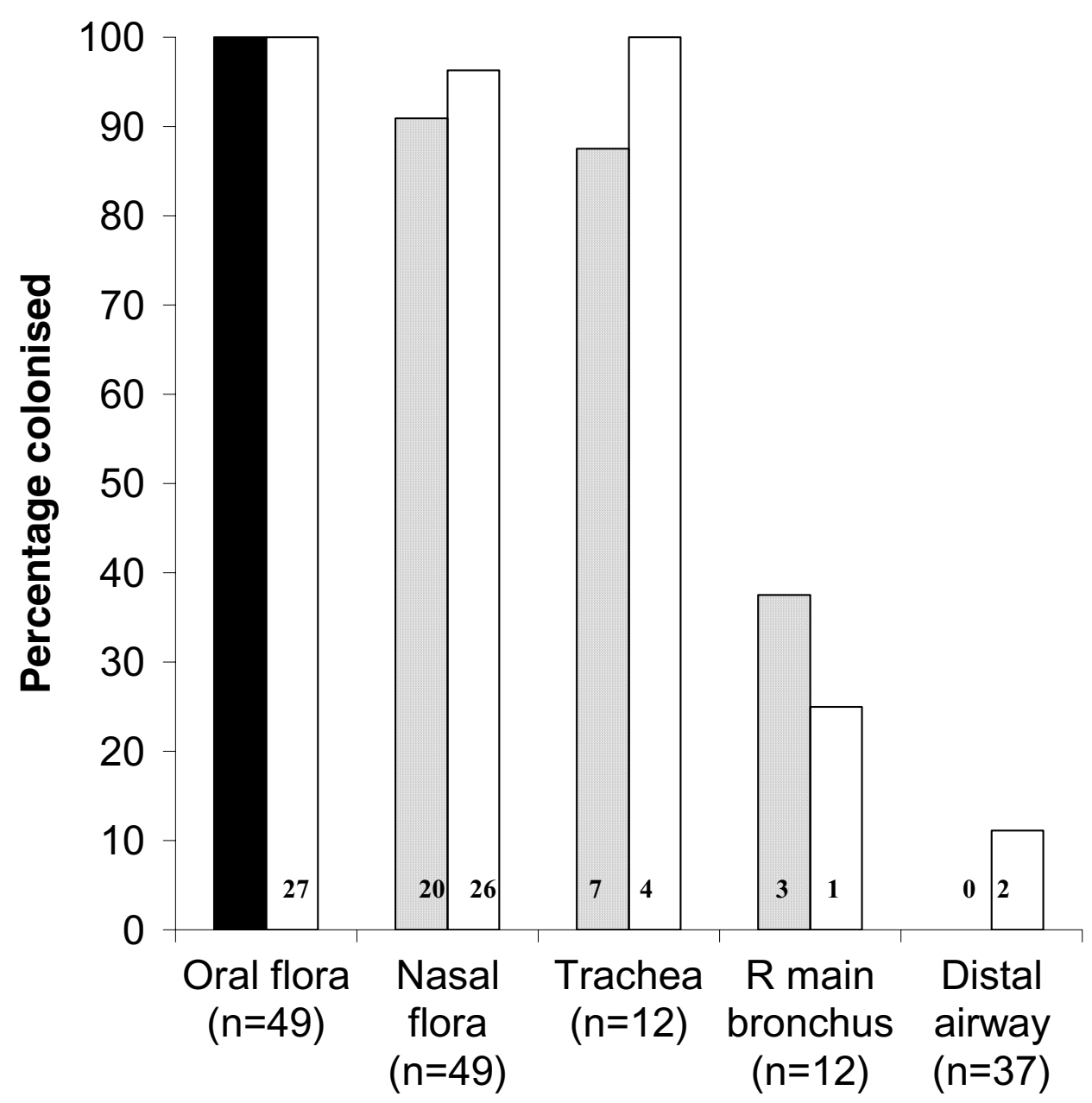

Figure I

Bacterial colonisation of the airways by HIV status. The figure shows the percentage of adults with bacterial colonisation of the oro-pharynx, posterior nasopharynx, trachea, right main bronchus and distal bronchi as measured using swabs (pharynx), tracheal washing and protected specimen brushing (right main bronchus and distal bronchi). Subjects are grouped according to HIV status and show no significant difference in percent colonisation at any site between HIV infected and nonHIV infected adults. The actual numbers of isolates are indicated over the bars and the bacteriological detail is included in the text.

bronchus PSB of one HIV positive subject (CD4 count of 125 cells/ul) in whom Aspergillus spp. was recovered from tracheal washings.

\section{Discussion}

Using the PSB method, we obtained lower respiratory tract samples from HIV-infected and HIV uninfected adults. There were no bacteria recovered from the most distal airways of HIV infected adults, and equal numbers of bacteria in the right main bronchus of HIV infected and uninfected adults. Significant numbers of common nasal colonizing organisms were recovered from the proximal airways of HIV infected and uninfected adults. No relation was observed between bacterial colonisation and CD4 count. There was therefore no evidence of increased bacterial colonisation in the bronchi of HIV infected adults.

The PSB method may underestimate bacterial colonisation due to the small volume of sample collected. Our methods detected sufficient organisms in upper respiratory sites, however, that we are confident of the sterile results reported in the lower airway. 
HIV infected adults have at least 10 times the incidence of bacterial pneumonia reported for age-matched non-HIVinfected adults $[1,2]$. Using the rate of bacterial detection observed in the lower respiratory tract in non-HIVinfected adults (2/18), our hypothesis was that most HIV infected subjects might have an alteration of tracheobronchial flora as is the case in bed-bound patients [3]. This study had $90 \%$ power to show a 6 or more times increase in the rate of bacterial recovery from the lower respiratory tract of HIV infected adults $(\mathrm{p}=0.05)$ but in the event there were no bacteria at all recovered from this site in the HIV infected group. We therefore reject the hypothesis that the increased rate of pneumonia seen in HIV infected adults might be due to increased colonization of the lower respiratory tract.

An important caveat of this finding, however, is that the colonisation of distal airways by Streptococcus pneumoniae was noted in only one subject and only as far as the right main bronchus. It is possible that HIV infected adults experience an increased rate of pneumococcal colonisation in the distal airways but the 6 patients with nasopharyngeal pneumococcal carriage in this study are insufficient to test this alternative hypothesis. In conclusion, therefore, there was no evidence of increased bacterial colonisation of the lower respiratory tract. Alternative explanations of the excess pneumococcal disease seen in otherwise healthy HIV infected patients must be sought, and vaccine strategies designed to enhance immune defences against the pattern of airway flora described in this study.

\section{Competing interests}

None declared.

\section{Authors' contributions}

SB Gordon: planned and drafted the design of the study, carried out all bronchoscopy and PSB procedures, analysed data and drafted the paper.

J Musaya: collected nasopharyngeal samples, carried out bench microbiological diagnosis and collated data.

L Wilson: helped in study design, supervised microbiology, analysed data and contributed to paper writing.

A Phiri: processed samples at microbiology bench.

EE Zijlstra: approved study design and contributed to manuscript writing.

ME Molyneux: approved study design and contributed to manuscript writing

\section{Acknowledgements}

The authors would like to thank their volunteers and the staff of the Queen Elizabeth Central Hospital, Blantyre, Malawi for their willing cooperation with this study. We also thank the bronchoscopy suite and laboratory staff of the Wellcome Trust Research Laboratories, Blantyre, Malawi for technical assistance and Dr Neil French for reviewing all the chest radiographs and making helpful comments on the draft manuscript. This work received financial support from the Wellcome Trust of Great Britain. SG holds a Wellcome Trust Career Development Fellowship; MEM is a Director of the Liverpool Wellcome Trust Centre for Clinical Tropical Research and this work forms part of the Malawi-Liverpool-Wellcome Trust Programme of Research in Clinical Tropical Medicine.

\section{References}

I. Hirschtick RE, Glassroth J, Jordan MC, Wilcosky TC, Wallace JM, Kvale PA, Markowitz N, Rosen MJ, Mangura BT, Hopewell PC: Bacterial pneumonia in persons infected with the human immunodeficiency virus. Pulmonary Complications of HIV Infection Study Group. N Engl J Med 1995, 333:845-85I.

2. Gilks CF, Ojoo SA, Ojoo JC, Brindle RJ, Paul J, Batchelor BI, Kimari JN, Newnham R, Bwayo J, Plummer FA, Warrell DA: Invasive pneumococcal disease in a cohort of predominantly HIV-I infected female sex-workers in Nairobi, Kenya. Lancet 1996, 347:718-723.

3. Rello J, Cabello H, Torres A: Epidemiology, risk and prognostic factors of nosocomial pneumonia. Volume 2. Ist edition. Edited by: TorresA and WoodheadM. European Respiratory Society; 1997:82-100.

4. Monso E, Ruiz J, Rosell A, Manterola J, Fiz J, Morera J, Ausina V: Bacterial infection in chronic obstructive pulmonary disease. A study of stable and exacerbated outpatients using the protected specimen brush. Am J Respir Crit Care Med 1995, 152:1316-1320.

\section{Pre-publication history}

The pre-publication history for this paper can be accessed here:

http://www.biomedcentral.com/1471-2334/3/29/prepub

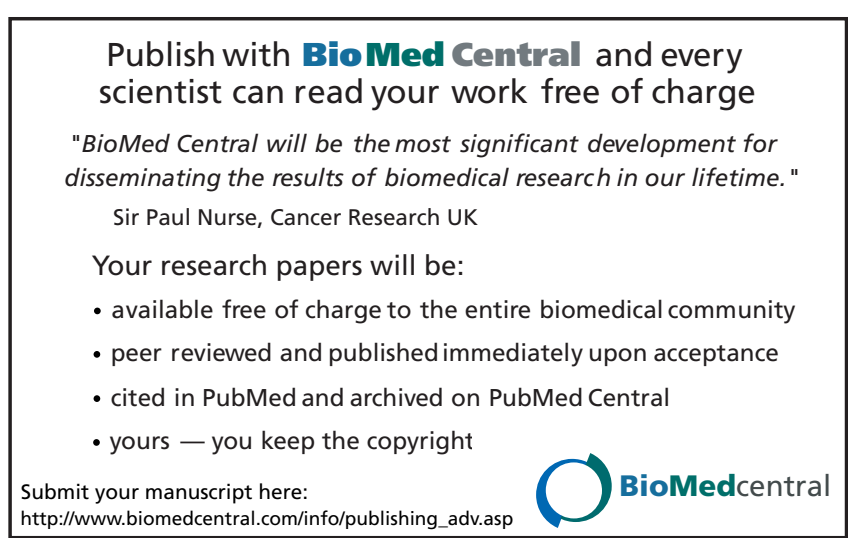

